\title{
Cartilage Palisade Type 1 Tympanoplasty: Anatomical and Audiological Outcomes
}

\author{
${ }^{1}$ Shweta Gupta, ${ }^{2}$ Himani Lade, ${ }^{3}$ Deepak Verma, ${ }^{4}$ Noor UI Din Malik
}

\begin{abstract}
Objectives: To evaluate the anatomical (an intact tympanic membrane without atelectasis or lateralization) and audiological outcomes of type 1 cartilage palisade tympanoplasty.

Methods: The prospective study was done at a tertiary referral institute included 30 patients with mucosal type chronic otitis media requiring type 1 tympanoplasty including subtotal or total perforations and revision cases. The tympanic membrane reconstruction was done using full thickness broad cartilage palisades harvested from the tragus or concha with attached perichondrium laterally. Patients were assessed at 1st, 3rd and 6th postoperative months for assessment of graft uptake, healing of tympanic membrane and hearing evaluation using pure tone audiometry.
\end{abstract}

Results: Total 27 out of 30 patients had fully epithelized completely healed grafts postoperatively at 1 month (success rate of $90 \%$ ) while three patients had small defect. One out of these three patients showed complete healing of graft while remaining two had persistent defect at 3rd and 6th months postoperatively. The mean pure tone air bone gap considerably reduced from $33.27 \pm 4.29 \mathrm{~dB}$ preoperatively to $12.67 \pm 5.68 \mathrm{~dB}$ postoperatively at 6 months.

Conclusion: Tympanic membrane reconstruction using full thickness palisades of cartilage provides good anatomical and audiological results with significant improvement of hearing specially in cases of subtotal or total perforations, revision surgery, atelectasis and Eustachian tube disorders where healing of tympanic membrane has much poorer prognosis irrespective of the surgical technique used.

Keywords: Cartilage, Palisade, Tympanoplasty.

How to cite this article: Gupta S, Lade H, Verma D, Malik NUD. Cartilage Palisade Type 1 Tympanoplasty: Anatomical and Audiological Outcomes. Int J Otorhinolaryngol Clin 2015;7(3):117-120.

\section{Source of support: Nil}

Conflict of interest: None

\section{INTRODUCTION}

Tympanoplasty is the surgical procedure performed for the reconstruction of ear drum and/or the ossicles. Since

\section{$1,3,4$ Resident, ${ }^{2}$ Consultant \\ ${ }^{1-4}$ Department of ENT, VMMC and Safdarjang Hospital, New Delhi, India}

Corresponding Author: Deepak Verma, Resident, Department of ENT, VMMC and Safdarjang Hospital, New Delhi, India Phone: 01263210111, e-mail: dpkvrm20@gmail.com the introduction of tympanoplasty by Wullstein ${ }^{1}$ in 1952 and Zoellner ${ }^{2}$ in 1955, different types of graft materials have been used to reconstruct the tympanic membrane. These include: temporalis fascia, perichondrium, cartilage, periosteum, vein, dura mater, skin, fat, etc. Temporalis fascia remains the most commonly used material for tympanic membrane reconstruction in primary tympanoplasties because of the advantages that it can be obtained through the same post-aural incision used for tympanoplasties, available in sufficient quantity, basal metabolic rate and thickness almost equal to tympanic membrane. ${ }^{3}$

However, the main disadvantages of using fascia graft include the high degrees of failure in atelectatic ear, retraction pocket, subtotal or total perforations and eustachian tube dysfunction. Fascia graft also composed of irregularly arranged elastic fibers and fibrous connective tissue due to which its postoperative dimensions are unpredictable while cartilage has constant shape, firmer than fascia and also lack fibrous tissue, so that its postoperative dimensions remain the same, and therefore, perichondrium and/or cartilage grafts are used with good results. ${ }^{4}$ Cartilage is being used in place of temporalis fascia as a grafting material, especially for repairing the large perforations, scutum defects, for preventing or correcting the failure of previous procedures associated with chronic tubal dysfunction, atelectatic ear drum and for enhancing the biocompatibility of ossicular prosthesis with tympanic membrane. ${ }^{5}$ The various techniques of cartilage tympanoplasty include perichondrium/cartilage island flap, cartilage shield techniques, palisade technique and inlay butterfly graft technique. ${ }^{6}$ Cartilage is well-nourished by diffusion, show great adaptation with tympanic membrane, provides firm support to prevent retraction and can resist deformation from pressure variation..$^{7-10}$ The various cartilages used are tragal, conchal, triangular fossa and septal cartilage. However, the disadvantages of cartilage grafts are that they mechanically reduce the vibratory pattern of tympanic membrane, contributing to some impairment in functional results, especially in higher tones and also create an opaque tympanic membrane which could hide a residual cholesteatoma. ${ }^{11}$

Since, cartilage grafts are gaining more popularity in the reconstruction of middle ear ossicles and/or ear drum 
in the last few years. The current study was undertaken to observe the anatomical and functional outcomes of palisade cartilage tympanoplasty.

\section{METHODS}

Prospective review at a tertiary referral center between 2013 and 2015 included 30 patients with mucosal type chronic otitis media requiring type 1 tympanoplasty including subtotal or total perforations and revision cases which has been dry for the previous 4 weeks and normal middle ear mucosa while the patients with small or moderate sized perforations, discharging ears and with active squamosal disease were excluded. The tympanic membrane reconstruction was done using full thickness broad cartilage palisades harvested from the tragus or concha with attached perichondrium laterally.

A written informed consent was obtained from all patients before surgery explaining the procedure, possible outcomes and complications. Patients were informed of the probability of postaural or endaural incisions. Institutional Ethics Committee clearance was obtained for the study.

Preoperative and postoperative hearing assessment was done using pure tone audiometry and averages were calculated as mean of frequencies 500, 1000, 2000 and $4000 \mathrm{~Hz}$.

\section{Surgical Technique}

All the subjects underwent the said surgical procedure under local/general anesthesia using the postauricular/ permeatal approach. A $1.5 \times 2 \mathrm{~cm}$ piece of cartilage was harvested from either the tragus or the cymba conchae and perichondrium was removed from one side and preserved laterally. The harvested graft was sectioned into palisades of about 0.5 to $1 \mathrm{~mm}$ width. Tympanomeatal flap was elevated after freshening the margins of the perforation and ossicular chain was inspected. The two semilunar palisades were placed in the tympanic cavity as underlay grafts, first anteriorly, and then posteriorly. The small palisade was placed along the malleus handle or as a prolongation of malleus handle (Fig. 1). The external auditory canal was packed with gelfoam and the incision sutured.

Harvesting of conchal cartilage: William Wilde's post-aural incision was given by starting at the highest attachment of the pinna, following the curve of retro-auricular groove and ending at the mastoid tip. Blunt dissection used to expose the conchal cartilage with overlying perichondrium intact and attached to graft. The index finger was placed in the conchal fossa laterally, guiding the knife medially to cut conchal cartilage leaving an adequate rim along the fossa. The entire conchal fossa cartilage except that needed to support ear was removed (Fig. 2).

Harvesting of tragal cartilage: A $15 \mathrm{~mm}$ long incision was made 2 to $3 \mathrm{~mm}$ below the dome of tragal cartilage in one sweep through the skin, posterior perichondrium, cartilage and anterior perichondrium. Extraperichondrial plane was created on both sides and cartilage graft was excised with perichondrium intact on both sides (Fig. 2).

\section{Parameters Assessed}

Graft uptake and failure: Graft was considered 'taken-up' if no perforation was noted at 1st, 3rd and 6th postoperative month, and considered failure if residual or recurrent perforation was noted at above mentioned time. A successful graft was defined as one which was well healed without any perforation/retraction/atelectasis or lateralization even at 6 months.

Postoperative hearing: Postoperative air bone gap, bone conduction threshold and air bone gap closure were considered as the objective method to assess and compare the hearing improvement.
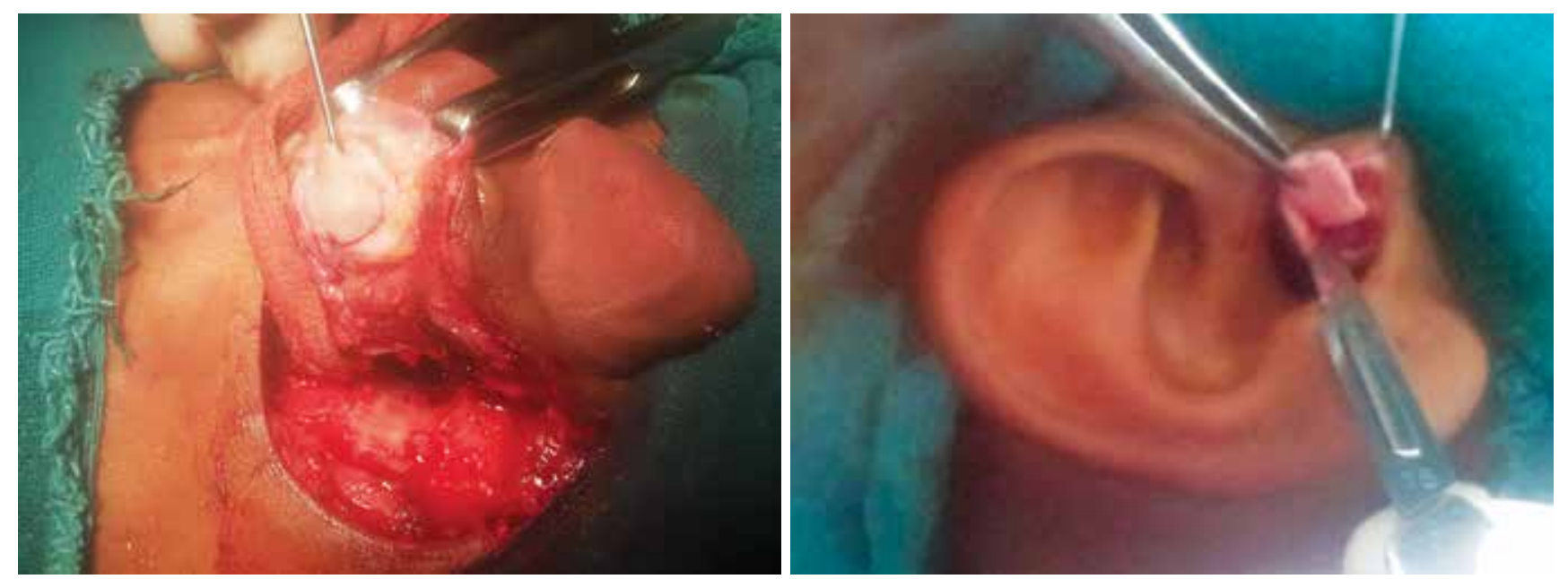

Fig. 1: Harvesting of conchal and tragal cartilage 

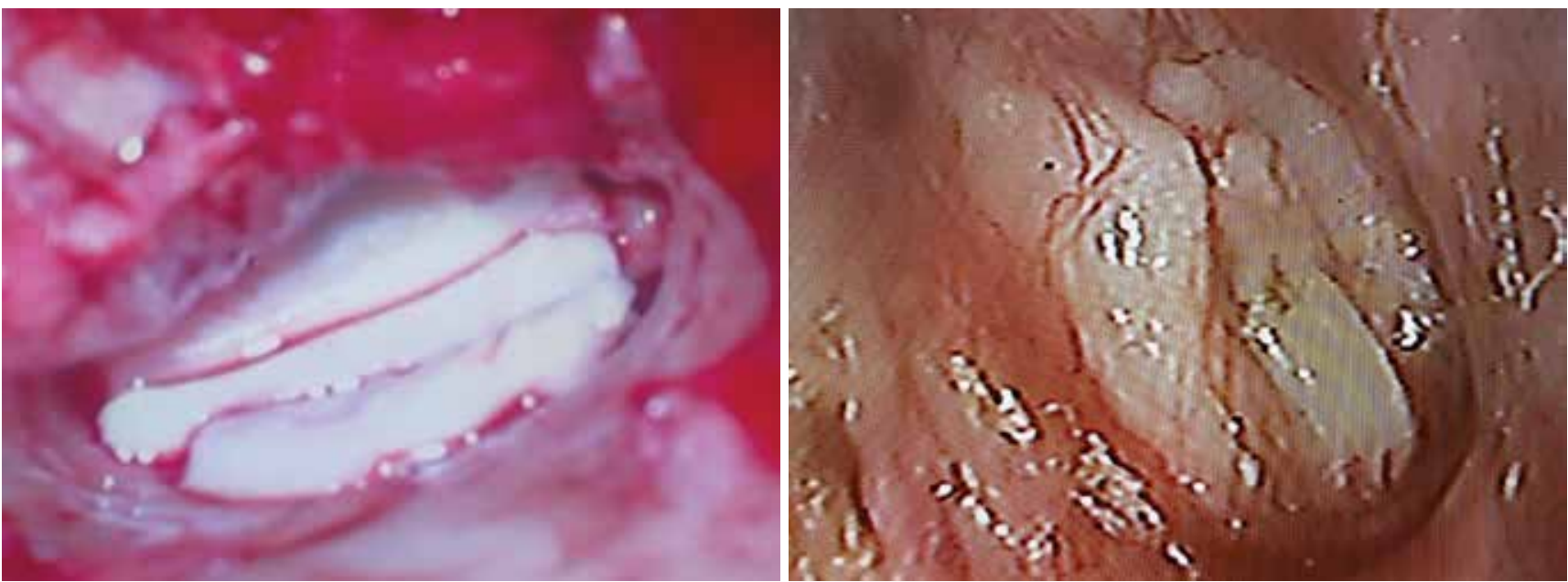

Fig. 2: Intraoperative placement of cartilage palisades and postoperative (3rd month) showing thin leash of blood vessels

\section{Results}

In our study, the mean age of patients was $33.70 \pm 9.96$ years with $70 \%$ females $(21 / 30)$ and $30 \%$ males $(9 / 30)$. Total $28(93.3 \%)$ out of 30 patients were primary cases while remaining two $(6.7 \%)$ underwent revision tympanoplasty. Among the study population five $(16.7 \%)$ patients had large perforation while 25 exhibited (83.3\%) subtotal perforation. Total 27 out of 30 patients had fully epithelized and completely healed grafts postoperatively at 1 month (success rate of $90 \%$ ) while three patients had small defect. One out of these three patients with subtotal perforation showed complete healing of graft while remaining two with large perforation had persistent defect at 6th month postoperatively (Graph 1). The mean pure tone air bone gap considerably reduced from $33.27 \pm 4.29$ $\mathrm{dB}$ preoperatively to $12.67 \pm 5.68 \mathrm{~dB}$ postoperatively at 6 months (Graph 2).

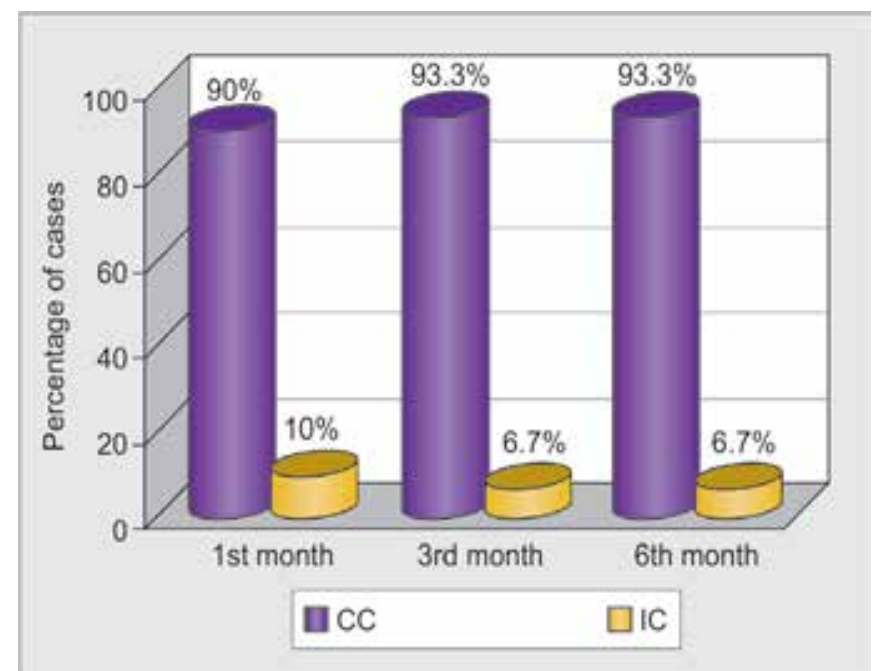

Graph 1: Comparison of status of defect at 1st, 3rd and 6th postoperative month (CC: Complete closure; IC: Incomplete closure)

\section{DISCUSSION}

Cartilage is a suitable graft material in tympanoplasty for advanced ear pathology which along with tympanic membrane, reconstruction of other structures of cavum tympani also required. Cartilage properties, unlike fascia or perichondrium are significantly distinctive. It can be shaped into fragments of various shapes, sizes and thicknesses, with perichondrium on one or both sides. By placing these fragments in various ways and various positions, middle ear reconstruction can be performed successfully. Cartilage as a graft material resists negative middle ear pressure to a much greater degree than fascia due to its rigidity which has been well-documented by laser Doppler vibrometric studies and also seems to offer more resistance to infection and lack of vascularization, often seen in atrophic drums and revision surgery. ${ }^{12}$

In our study, $12(40 \%)$ out of 30 patients had disease restricted to one ear while $18(60 \%)$ had bilateral ear disease. Many authors concluded that good Eustachian

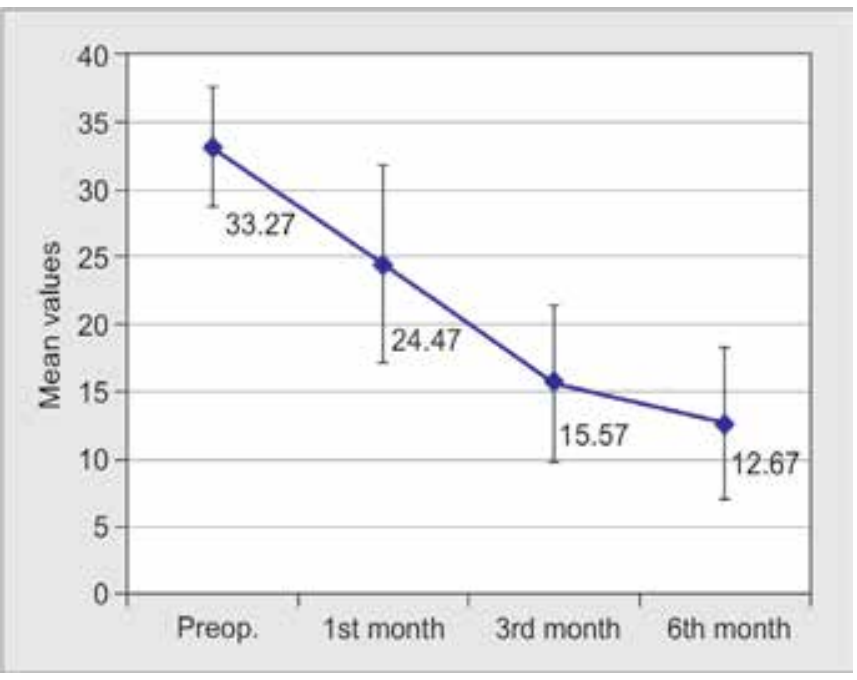

Graph 2: Distribution of mean preoperative and postoperative air bone gap at 1st, 3rd and 6th postoperative months 
tube function in the contralateral ear predicted good surgical outcome, while poor Eustachian function was not necessarily an indicator of poor outcome. They have used contralateral tympanic membrane perforation, retraction or effusion to be indications of poor Eustachian tube function. Onal et $\mathrm{al}^{13}$ in their study concluded that in bilateral chronic otitis media cartilage tympanoplasty gives better results as compared to temporalis fascia graft. In our study, five (16.7\%) patients had large perforation and 25 (83.3\%) patients had subtotal perforation. In Ozbek ${ }^{14}$ et al study, all the patients had perforation of size $>50 \%$ of tympanic membrane and similiarly Cagdas Kazikdas ${ }^{15}$ et al conducted a study with subtotal perforation using palisade cartilage grafts and temporalis fascia grafts and concluded that palisade cartilage might be recommended as a method of choice in case of subtotal or total perforations.

Graft take up rate was assessed by the complete closure of tympanic membrane perforations at the end of $1 \mathrm{st}, 3 \mathrm{rd}$ and 6th postoperative months respectively. In our study, 27 out of 30 patients had fully epithelized and completely healed grafts postoperatively at 1 month (success rate of $90 \%$ ) while three patients had small defect. One out of these three patients with subtotal perforation showed complete healing of graft while remaining two with large perforation had persistent defect at 6th month postoperatively. The mean pure tone air bone gap pre- and postoperatively was $33.27 \pm 4.29 \mathrm{~dB}$ and $12.67 \pm 5.68 \mathrm{~dB}$ respectively. Boone ${ }^{16}$ et al conducted a study and observed that successful closure without reperforation was obtained in 90 out of 95 patients $(94.7 \%)$. Average postoperative puretone average air bone gap was $12.2 \pm 7.3 \mathrm{~dB}$ compared with $24.6 \pm 13.8 \mathrm{~dB}$ preoperatively $(\mathrm{p}<0.001)$. The results of all these studies are comparable with our results.

\section{CONCLUSION AND RECOMMENDATIONS}

Tympanic membrane reconstruction using full thickness palisades of cartilage provides good anatomical and audiological results with significant improvement of hearing specially in cases of revision surgery, atelectasis, eustachian tube disorders and subtotal or large perforations where healing of tympanic membrane has much poorer prognosis irrespective of the surgical technique used. Good graft uptake results of cartilage palisade grafts are related to nourishment of cartilage grafts by diffusion and merging of graft with tympanic membrane effectively and showing great adaptation with tympanic membrane.

\section{REFERENCES}

1. Wullstein HL. Functional operations in the middle ear with split thickness skin graft. Arch Otorhinolaryngol 1953;161: 422-435.

2. Zoellner F. The principles of plastic surgery of the sound conducting apparatus. J Laryngol Otol 1955;69:567-569.

3. Heermann H. Tympanic membrane plastic with temporal fascia. Hals-Naser-Ohren 1960;9:136-139.

4. Heermann JJ, Heermann H, Kopstein E. Fascia and cartilage palisade tympanoplasty: nine years experience. Arch Otolaryngol 1970;91(3):228-241.

5. Neumann A, Hennig A, Schultzcoulon HJ. Morphological and functional results of palisade cartilage tympanoplasty. HNO 2002;50(10):935-939.

6. Tos M. Cartilage tympanoplasty methods: proposal of a classification. Otolaryngol Head Neck Surg 2008;139(6): 747-758.

7. Amedee RG, Mann WJ, Riechelmann H. Cartilage palisade tympanoplasty. Am J Otol 1989;10(6):447-450.

8. Neumann A, Schultzcoulon HJ, Jahnke K. Type III tympanoplasty applying the palisade cartilage technique: a study of 61 cases. Otol Neurotol 2002;24(1):33-37.

9. Velepic M, Bonifacic M, Manestar D, Bonifacic D. Cartilage palisade tympanoplasty and diving. Otol Neurotol 2001;22(4): 430-432.

10. Uzun C, Caye P, Andersen J, Tos M. A tympanometric comparison of tympanoplasty with cartilage palisades or fascia after surgery for tensa cholesteatoma in children. Laryngoscope 2003;113(10):1751-1757.

11. Uzun C, Yagiz R, Tas A, Adali MK, Koten M, Karasalihoglu AR. Combined Heermann and Tos technique in cholesteatoma surgery: surgical technique and preliminary results. J Laryngol Otol 2005;119(6):429-435.

12. Bernal-Sprekelsen M, Romaguera Lilso, Sanz Gonzalo JJ. Cartilage palisades in type III tympanoplasty: anatomical and functional long-term results. Otol Neurotol 2003;24(1): 38-42.

13. Onal K, Arslanoglu S, Oncel S, Songu M, Kopar A, Demiray U. Perichondrium/Cartilage island flap and temporalis muscle fascia in type I tympanoplasty. J Otolaryngol Head Neck Surg 2011;40(4):295-299.

14. Ozbek C, Çifti O, Tuna EEU, Yazkan O, Ozdem C. A comparison of cartilage palisades and fascia in type 1 tympanoplasty in children: anatomic and functional results. Otol Neurotol 2008;29:679-683.

15. Cagdas Kazikdas K, Boyraz I, Karabulut E. Palisade cartilage tympanoplasty for management of subtotal perforations. Eur Arch Otorhinolaryngol 2007;264(9):985-989.

16. Boone RT, Gardner EK, Dornhoffer JL. Success of cartilage grafting in revision tympanoplasty without mastoidectomy. Otol Neurotol 2004;25(5):678-681. 